\title{
Über die Struktur der Lösung des Mehrelektronenproblems im Festkörper und ein Theorem von Bloch
}

\author{
Von Hermann Haken \\ Aus dem Institut für theoretische Physik der Universität Erlangen \\ (Z. Naturforschg. 9a, 228-235 [1954]; eingegangen am 16. Dezember 1953)
}

\begin{abstract}
Der Untersuchung wird eine Schrödinger-Gleichung für die Elektronen zugrunde gelegt, in die abstands-, geschwindigkeits- und spinabhängige Wechselwirkungen aufgenommen sind. Die Elektronen sollen sich außerdem in einem Gitter bewegen, dessen periodische Struktur durch Gitterschwingungen deformiert werden kann. Die Gestalt des Hamilton-Operators, der alle diese Wechselwirkungen enthält, wird diskutiert. Die Form der Lösung für das Gesamtsystem, bestehend aus Elektronen und Schallquanten, ergibt sich zu $e^{i \Re \Re} U_{\Re}\left(\Re, \mathfrak{r}_{i}-\mathfrak{r}_{j} ; b_{\lambda}^{*}\right)$. Die Funktion $U_{\Re}$ hängt darin noch in ganz bestimmter, näher zu erläuternder Weise von der Schwerpunktskoordinate $\Re$ und den Relativkoordinaten $\mathfrak{r}_{i}-\mathfrak{r}_{j}$ der Elektronen sowie von den Erzeugungsoperatoren $b_{\lambda}^{*}$ der Schallquanten ab. Der Erwartungswert des Elektronenstroms ist mit der Energie durch die in Strenge gültige Formel $e \overline{\mathfrak{v}}=e n / \hbar \operatorname{grad}{ }_{\mathfrak{H}} E$ verknüpft. Daraus ergibt sich insbesondere der allgemeine Beweis für das Theorem von Bloch, daß der energetisch tiefste Zustand des Festkörpers stromlos ist. Auf die Notwendigkeit einer Neuformulierung der Theorie der Leitfähigkeit wird hingewiesen.
\end{abstract}

$\mathrm{D}$ ie heutige Elektronentheorie des Festkörpers beruht im wesentlichen auf dem Bändermodell. Obgleich die Voraussetzungen dieses Modells — die Elektronen sollen sich ohne jede gegenseitige Wechselwirkung in einem streng periodischen, zeitlich konstanten Potentialfeld bewegen - in der Natur keineswegs erfüllt sind, so vermittelt es doch für wichtige Eigenschaften des Festkörpers ein grundsätzliches Verständnis, etwa für die Unterscheidung von Leitern und Nichtleitern und das Auftreten der Defektelektronen. Außerdem erweist sich dieses Modell so anpassungsfähig, daß es gerade auch zur Behandlung von Effekten geeignet ist, die durch die naturgegebenen Abweichungen gegenüber den Voraussetzungen des Modells entstehen, beispielsweise zur Behandlung des elektrischen Widerstandes als Folge der Wechselwirkung der Elektronen mit den Gitterschwingungen. Wesentlich bei dieser Behandlungsweise ist, daß die Abweichungen in irgendeinem, jeweils näher zu präzisierenden Sinne als „,kleine Störungen“ aufgefaßt werden können. Diese Voraussetzung ist jedoch nicht immer erfüllt und es gibt tatsächlich eine ganze Reihe experimenteller Befunde, für deren theoretische Behandlung das Bändermodell keinen geeigneten Ausgangspunkt darstellt. Als Beispiele nennen wir die (von Schott ky so bezeichneten) Offenband-Halbleiter, die auf Grund des Bändermodells ein offenes Band haben sollten, also Leiter sein müßten, oder besonders die
Erscheinung des Ferromagnetismus. In beiden Fällen kommt es auf die feineren Züge der Coulombschen Wechselwirkung zwischen den Elektronen an, die durch eine pauschale Potentialangabe nicht mehr zu erfassen sind. Es sind daher eine Reihe andersartiger Näherungen, beispielsweise die von Heitler-London, herangezogen worden, die die Coulombsche Wechselwirkung in einem höheren Maße berücksichtigen, als es das Bändermodell tun kann. In einer Arbeit von Volz und Haken ${ }^{1}$ wurde die Frage aufgegriffen, welche exakt gültigen Aussagen sich über die Wellenfunktion machen lassen, wenn in den Ansatz für die Schrödinger-Gleichung von vornherein auch die Coulombsche Wechselwirkung zwischen den Elektronen explizit mit aufgenommen wird. Wie sich dabei ergab, stehen die Aussagen über die Gestalt der Wellenfunktion, das Verhalten unter einem äußeren elektrischen Feld usw. in enger Analogie zu denen des Bändermodells.

Es gibt nun aber noch einen zweiten Bereich von Problemen, die ebenfalls im Rahmen des Bändermodells nicht behandelt werden können. Wir denken hier an solche Fälle, in denen die Wechselwirkung zwischen den Elektronen und Gitterschwingungen sich nicht in der üblichen Weise mit Hilfe der Störungsrechnung behandeln läßt. Hierzu gehören bei-

${ }^{1}$ H. Volz u. H. Haken, Z. phys. Chem. 198, 61 [1951]. 
spielsweise die Bewegung einzelner Elektronen in polaren Medien, wahrscheinlich auch das Widerstandsverhalten von Gold bei tiefen Temperaturen sowie die noch nicht völlig geklärte Rolle der ,„Umklapp-Prozesse".

Ein Gebiet schließlich, das die Berücksichtigung aller Arten von Wechselwirkungen zwischen den Elektronen untereinander und zwischen den Elektronen und Gitterschwingungen notwendig machen dürfte, ist das der Supraleitung. Hier ist es bis heute noch nicht entschieden, welche Art von Wechselwirkung für diese Erscheinung verantwortlich zu machen ist, ja nicht einmal, ob überhaupt eine einzige Wechselwirkung zur Erklärung ausreicht. Hierzu kommt noch, daß die Störungsrechnung zur Behandlung dieses Problems nicht geeignet ist ${ }^{2}$.

Aufgabe dieser Arbeit soll es daher sein, von vornherein sämtliche Wechselwirkungsarten in den Ansatz für die Schrödinger-Gleichung mit aufzunehmen und zu untersuchen, welche allgemeinen und exakt gültigen Aussagen sich dann noch machen lassen. Es wird sich zeigen, daß die hier erhaltenen Aussagen formal aufs engste mit denen des Bändermodells übereinstimmen, sich aber inhaltlich davon - wie an Modellbeispielen in nachfolgenden Arbeiten gezeigt werden soll - ganz wesentlich unterscheiden können.

\section{Die Gestalt des Hamilton-Operators}

Eine Gleichung, welche die Wechselwirkung zwischen den Elektronen bis zu einer beliebig vorgegebenen Genauigkeit beschreibt, gibt es heute noch nicht. Das Problem, eine solche Gleichung explizit aufzustellen, das aufs engste mit der Frage der gebundenen Zustände in der Quantenelektrodynamik verbunden ist, soll uns hier indessen nicht berühren. Wir nehmen lediglich an, daß sich das Geschehen durch eine Schrödinger-Gleichung der Form

$$
\mathscr{H} \psi=-\frac{\hbar}{i} \frac{\partial}{\partial t} \psi
$$

bzw. im stationären Fall durch

$$
\mathscr{H} \psi=E \psi
$$

beschreiben läßt, da bei den geringen Energie-Umsetzungen im Festkörper der Hamilton-Formalismus wohl nicht abgeändert zu werden braucht.

${ }^{2}$ M. R. Schafroth, Helv. Phys. Acta 24, 645 [1951]; Nuovo Cim. 9, 291 [1952].
Wir betrachten im folgenden ein System von $n$ Elektronen, die die Raumkoordinaten $\mathfrak{r}_{1}, \mathfrak{r}_{2}, \ldots, \mathfrak{r}_{n}$ haben. Je nach Art der zugrunde gelegten Genauigkeit sind den Elektronen auch Spinkoordinaten zuzuschreiben bzw. ist die Wellenfunktion aus Spinoren aufzubauen. Bewegen sich die Elektronen in einem Raum mit konstantem Potential, so ist kein Raumpunkt ausgezeichnet; der Hamilton-Operator kann daher nur von den Relativkoordinaten der Elektronen und den Ableitungen $\partial / \partial x_{1}, \partial / \partial y_{1}, \partial / \partial z_{1}, \ldots$ abhängen, hat also die Form

$$
\mathscr{H}\left(\frac{\partial}{\partial x_{1}}, \frac{\partial}{\partial y_{1}}, \frac{\partial}{\partial z_{1}}, \ldots ; \mathfrak{r}_{i}-\mathfrak{r}_{j}\right) .
$$

Außerdem kann $\mathscr{H}$ noch Funktion von Spinoperatoren bzw. Spinmatrizen sein.

Beim Festkörper haben wir es nun nicht nur mit Elektronen, sondern auch mit den Gitterrümpfen bzw. mit den Kernen zu tun. Wir müßten daher eigentlich eine Gleichung ansetzen, die auch die Koordinaten und Impulse der Kerne umfaßt und die Wechselwirkung zwischen allen Bestandteilen des Systems beschreibt. Eine solche Problemstellung würde jedoch viel zu viel hier gar nicht interessierende Spezialfälle enthalten, so daß die sich dann ergebenden allgemeinen Aussagen ziemlich bedeutungslos wären. Im Anschluß an das übliche Vorgehen in der Elektronentheorie des Festkörpers machen wir deshalb von der Erfahrungstatsache Gebrauch, daß die Atomrümpfe schon zu einem Gitter zusammengetreten sind und um ihre Gleichgewichtslage nur noch kleine Schwingungen ausführen.

Das Gitter, das wir für das folgende stets als Translationsgitter voraussetzen wollen, sei zunächst in Ruhe. Der Hamilton-Operator in Gl. (2), der sich in diesem Falle weiterhin nur auf die Elektronen bezieht, wird nun auch eine Funktion der Einzelkoordinaten. Auf jeden Fall können wir aber feststellen, daß der Hamilton-Operator in sich übergeht, wenn wir alle Elektronenkoordinaten gleichzeitig um einen der drei Gittervektoren $\mathfrak{a}_{1}, \mathfrak{a}_{2}, \mathfrak{a}_{3}$, die die Gitterzelle des Translationsgitters aufspannen, verschieben.

Durch die Berücksichtigung der Gitterschwingung ändert sich die Energie des Systems einmal um die Schwingungsenergie der Atomrümpfe (bzw. Kerne), dann aber auch durch die Verzerrung des vom Grundgitter herrührenden Potentialfeldes bei der Auslenkung der Atome. Zur Wiedergabe des ersten Anteils nehmen wir wie üblich an, daß die Aus- 
lenkungen der Atome sich aus harmonischen, den Kristall durchziehenden Wellen zusammensetzen lassen. Die Energie dieser „Normalschwingungen“ wird dann dargestellt durch einen Hamilton-Operator $\mathscr{H}_{\mathrm{Osz}}$, der additiv zu dem ursprünglichen Hamilton-Operator der Elektronen hinzutritt. Zur expliziten Formulierung von $\mathscr{H}_{\text {Osz. }}$ benutzen wir Operatoren ${ }^{3} b_{\lambda}^{*}$ und $b_{\lambda}$, die die Erzeugung und Vernichtung von „Schallquanten“, d.h. von Quanten dieser Normalschwingungen mit dem Index $\lambda$ beschreiben. Ist $\omega_{\lambda}$ die Frequenz ${ }^{4}$ einer solchen Schwingung, dann läßt sich schreiben ${ }^{5}$

$$
\mathscr{H}_{\mathrm{Osz} .}=\sum_{\lambda=1}^{N} \hbar \omega_{\lambda} b_{\lambda}^{*} b_{\lambda} .
$$

Es wird für das folgende genügen, diesen Anteil dadurch zu berücksichtigen, daß wir den neuen Hamilton-Operator in der Form

$$
\mathscr{H}=\mathscr{H}\left(\frac{\partial}{\partial x_{1}}, \frac{\partial}{\partial y_{1}}, \ldots ; \mathfrak{r}_{i}, b_{\lambda}^{*} b_{\lambda}\right)
$$

ansetzen.

Durch die Schwingungen der Rümpfe wird auch das Potential des Grundgitters, in dem sich die betrachteten Elektronen bewegen, dauernd verändert. Dies gibt Anlaß zu Zusammenstößen zwischen den Elektronen und Schallquanten. Ohne daß wir auf die genauere Form dieser Wechselwirkung einzugehen brauchen, können wir mit Hilfe der eben eingeführten Erzeugungs- und Vernichtungsoperatoren $b_{\lambda}^{*}, b_{\lambda}$ angeben, wie ein solches Wechselwirkungsglied aufgebaut ist: Wird ein Schallquant der Gitterwelle $\lambda$, die den Ausbreitungsvektor $\mathfrak{w}_{\lambda}$ besitzt, bei einem Zusammenstoß mit einem Elektron der Koordinate $\mathfrak{r}_{i}$ vernichtet, so wird dies durch das Glied

$$
b_{\lambda} e^{i \mathfrak{w} \mathfrak{w}_{i}}
$$

beschrieben. Diesem Einzelakt kommt eine Amplitude $W$ zu, die noch von den Elektronenkoordinaten, evtl. auch noch von den Impulsen abhängt. Da keine Gitterzelle vor der anderen ausgezeichnet ist, ist $W$ invariant gegenüber einer simultanen Verschiebung aller Elektronen um einen Gittervektor $\mathfrak{a}_{i}$. Wir werden für das folgende auch zulassen, daß schon im Ansatz Mehrfachprozesse vorkommen

${ }^{3}$ Wir benutzen die Darstellungsweise von R. Bekker u. G. Leibfried, Z. Phys. 125, 347 [1949].

${ }^{4}$ Auf die Frage, in welcher Weise die $\omega_{\lambda}$ zu bestimmen sind, und auf die damit verbundene Problematik gehen wir nicht ein. (S. hierzu: H. Fröhlich, Proc. können, daß also das Wechselwirkungsglied eine Potenzreihe in den Ausdrücken (5) wird. Ebenso finden natürlich Erzeugungsprozesse, die durch das zu (5) konjungiert komplexe Glied beschrieben werden, wie auch ,gemischte Prozesse“ statt. Der endgültige Hamilton-Operator hat daher die Gestalt

$$
\begin{aligned}
& \mathscr{H}=\mathscr{H}\left(\frac{\partial}{\partial x_{1}}, \frac{\partial}{\partial y_{1}}, \ldots ; \mathfrak{r}_{i} ;\right. \\
& \left.b_{\lambda}^{*} b_{\lambda} ; b_{\lambda} e^{i \mathfrak{w} \hat{\mathfrak{r}}_{i}}, b_{\lambda}^{*} e^{-i \mathfrak{w} \mathfrak{\mathfrak { r }}_{i}}\right) .
\end{aligned}
$$

Er hängt dabei von den allein im Argument stehenden Elektronenkoordinaten in der Weise ab, daß er bei einer gleichzeitigen Verschiebung aller dieser Elektronenkoordinaten um einen Vektor $\mathfrak{a}_{i}$ in sich übergeht.

Das wellenmechanische Problem ist erst vollständig definiert, wenn auch die Randbedingungen vorgegeben werden. Wir beschäftigen uns in dieser Arbeit mit dem Problem eines Kristalls, der in allen drei Dimensionen unendlich ausgedehnt ist. Dem steht die Tatsache gegenüber, da: $\beta$ wir nur endlich viele Elektronen, die gerade ein bestimmtes ,,Grundgebiet“ endlicher Größe neutralisieren sollen, in die Untersuchung mit aufgenommen haben. Die Frage, wie es trotzdem möglich ist, den Kristall insgesamt elektrisch neutral zu erhalten, ist in der zitierten Arbeit ${ }^{1}$ behandelt worden. Wie sich zeigt, wird dies in gewissem Sinne durch die Randbedingung erreicht, daß die Wellenfunktion periodisch in jeder einzelnen Elektronenkoordinate mit dem Grundgebiet (अ) ist. Diese Forderung wollen wir auch hier der Wellenfunktion auferlegen. Für die Gitterwellen verlangen wir ebenfalls die Periodizität mit dem gleichen Grundgebiet. Dadurch wird dann das gegenseitige Verhältnis der Term-Mannigfaltigkeit von Elektronen und Gitterschwingungen richtig wiedergegeben.

\section{Die Struktur der Lösung}

Die Schrödinger-Gleichung nehmen wir in der im vorigen Abschnitt abgeleiteten Form (6) an:

$$
\begin{aligned}
& \mathscr{H}\left(\frac{\partial}{\partial x_{1}}, \frac{\partial}{\partial y_{1}}, \ldots ; \mathfrak{r}_{i} ; b_{\lambda}^{*} b_{\lambda}\right. \\
&\left.b_{\lambda} e^{i \mathfrak{w}} \mathfrak{r}_{i}, b_{\lambda}^{*} e^{-i \mathfrak{w}_{\lambda} \mathfrak{r}_{i}}\right) \psi=E \psi .
\end{aligned}
$$

Die Lösung $\psi$ entwickeln wir nach den Eigenfunktionen der Gitteroszillatoren, also nach den Eigenfunktionen der Gleichungen

$$
\hbar\left(\omega_{i} b_{\lambda}^{*} b_{\lambda} \Phi=\varepsilon_{\varepsilon} \Phi\right.
$$

Roy. Soc. A 215, 291 [1952]; Y. Kitano und H. Nakano, Progr. Theor.Phys. 9, 370 [1953].)

${ }^{5} N$ ist die Zahl der Freiheitsgrade der $N / 3$ Kerne des ,,Grundgebiets“. Die konstante Nullpunktsenergie haben wir weggelassen. 
Diese Eigenfunktionen lassen sich in bekannter Weise (1. c. ${ }^{3}$ ) mit Hilfe der Erzeugungsoperatoren in $\operatorname{der}$ Form $^{6}$

$$
\varphi_{\nu_{1}, \nu_{2}, \ldots, \nu_{N}}=\left(b_{1}^{*}\right)^{\nu_{1}}\left(b_{2}^{*}\right)^{\nu_{2}} \ldots\left(b_{N}^{*}\right)^{\nu_{N}} \cdot \varphi_{0}
$$

darstellen, die angibt, daß der Oszillator 1 mit $v_{1}$ Quanten, der Oszillator 2 mit $v_{2}$ Quanten, ... besetzt ist. Der Ansatz für die gesamte Lösung $\psi$ lautet also

$\psi\left(\mathfrak{r}_{1}, \mathfrak{r}_{2}, \ldots, \mathfrak{r}_{n} ; b_{1}^{*}, \ldots, b_{N}^{*}\right)$

$=\sum_{(v)} v_{\nu_{1}, v_{2}, \ldots, v_{N}}\left(\mathrm{r}_{1}, \ldots, \mathrm{r}_{n}\right)\left(b_{1}^{*}\right)^{\nu_{1}} \ldots\left(b_{N}^{*}\right)^{v_{N}} \cdot \varphi_{0}$.

Die $v$ können darin, wie wir von Anfang an vereinbart hatten, auch noch von Spinkoordinaten bzw. Spinorindizes abhängen.

Wir ersetzen nun in Gl. (7) simultan $\mathfrak{r}_{i}$ durch $\mathfrak{r}_{i}+\mathfrak{a}$ und $b_{\lambda}^{*}, b_{\lambda}$ durch $b_{\lambda}^{*} e^{i \mathfrak{w} \lambda^{\mathfrak{a}}}, b_{\lambda} e^{-i \mathfrak{w} \lambda^{\mathfrak{a}}}$, wobei $\mathfrak{a}$ einer der drei die Gitterzelle aufspannenden Vektoren $\mathfrak{a}_{1}, \mathfrak{a}_{2}, \mathfrak{a}_{3}$ ist. Der Hamilton-Operator geht dabei in sich über. Die Wellenfunktion

$\psi\left(\mathfrak{r}_{1}+\mathfrak{a}, \mathfrak{r}_{2}+\mathfrak{a}, \ldots, \mathfrak{r}_{n}+\mathfrak{a} ;\right.$

$$
\left.b_{1}^{*} e^{i \mathfrak{w}_{1} \mathfrak{a}}, b_{2}^{*} e^{i \mathfrak{w}_{2} \mathfrak{a}}, \ldots, b_{N}^{*} e^{i \mathfrak{w}{ }_{N} \mathfrak{a}}\right)
$$

genügt wieder dieser Gleichung, muß sich also als Linearkombination der zum gleichen Eigenwert $E$ gehörigen Lösungen $\psi_{i}$, die linear unabhängig sein sollen, darstellen lassen. Dies gilt natürlich für alle Lösungen $\psi_{i}$, so daß wir das folgende Gleichungssystem erhalten

$$
\begin{aligned}
\psi_{i}\left(\mathfrak{r}_{1}+\mathfrak{a}, \ldots ; b_{1}^{*} e^{i \mathfrak{w}_{1} \mathfrak{a}}, \ldots\right) & \\
& =\sum_{j} c_{i j} \psi_{j}\left(\mathfrak{r}_{1}, \ldots ; b_{1}^{*}, \ldots\right) .
\end{aligned}
$$

Durch Einführung neuer Linearkombinationen $\widetilde{\psi}_{i}=\sum_{j} d_{i j} \psi_{j}$ können wir die Matrix $\left(c_{i j}\right)$ auf Hauptachsen bringen. Dies ist immer möglich, da die Transformationsmatrix keinen Einschränkungen unterliegt. (Die Frage nach der Orthogonalität der neuen Funktionen untersuchen wir im folgenden Abschnitt.) Man sieht übrigens leicht, daß die Matrix $\left(c_{i j}\right)$ auch eine Reziproke besitzt. Das Gleichungssystem erhält nach dieser Transformation die Form

$$
\psi_{i}\left(\mathfrak{r}_{1}+\mathfrak{a}, \ldots ; b_{1}^{*} e^{i \mathfrak{w}_{1} \mathfrak{a}}, \ldots\right)=\alpha_{i} \psi_{i}\left(\mathfrak{r}_{1}, \ldots ; b_{1}^{*}, \ldots\right) .
$$

Verschieben wir die Elektronengesamtheit um das $m$-fache eines Gittervektors, so multipliziert sich die Ausgangsfunktion mit einem Faktor $\alpha^{m}$. Damit die Wellenfunktion im Unendlichen beschränkt bleibt, muß daher $\alpha$ vom Betrage 1 sein, also die Form $e^{i L}$ haben. Aus Gründen, die gleich ersichtlich werden, spalten wir $\alpha$ in ein skalares Produkt aus dem Gittervektor $\mathfrak{a}$ und einem ,Wellenzahlvektor" $\Re$ auf, schreiben also

$$
\alpha=e^{i \Re \mathfrak{a} .}
$$

Wir ziehen nun die Entwicklung (9) heran und untersuchen, welche Aussagen sich über die Koeffizienten $v$ machen lassen:

$$
\begin{aligned}
\sum_{(v)} v_{\nu_{1}}, \ldots, \nu_{N} & \left(\mathfrak{r}_{1}+\mathfrak{a}, \ldots\right) \\
& \cdot e^{i \mathfrak{w}_{1} \nu_{1} \mathfrak{a}+i \mathfrak{w}_{2} \nu_{2} \mathfrak{a}+\ldots+i \mathfrak{w}_{N} \nu_{N} \mathfrak{a}}\left(b_{1}^{*}\right)^{\nu_{1}} \ldots\left(b_{N}^{*}\right)^{\nu_{N}} \varphi_{0} \\
= & e^{i \Re \mathfrak{a}} \sum_{(\nu)} v_{\nu_{1}, \ldots \nu_{N}}\left(\mathfrak{r}_{1}, \ldots\right)\left(b_{1}^{*}\right)^{\nu_{1}} \ldots\left(b_{N}^{*}\right)^{\nu_{N}} \varphi_{0} .
\end{aligned}
$$

Da verschiedene Eigenfunktionen $\left(b_{1}^{*}\right)^{\nu_{1}} \ldots\left(b_{N}^{*}\right)^{\nu_{N}} \varphi_{0}$ aufeinander orthogonal sind, gilt Gl. (11) auch schon für die einzelnen Koeffizienten

$$
\begin{gathered}
v_{\nu_{1}, \ldots, \nu_{N}}\left(\mathfrak{r}_{1}+\mathfrak{a}, \ldots\right) e^{i \mathfrak{w}_{1} \nu_{1} \mathfrak{a}+i \mathfrak{w} \mathfrak{w}_{2} \nu_{2} \mathfrak{a}+\ldots+i \mathfrak{i} \mathfrak{w}_{N} \nu_{N} \mathfrak{a}} \\
=e^{i \Re \mathfrak{r a}} v_{\nu_{1}, \ldots, v_{N}}\left(\mathfrak{r}_{1}, \ldots\right)
\end{gathered}
$$

Zur Lösung dieser Funktionalgleichung setzen wir

$$
\begin{aligned}
v_{\nu_{1}}, \ldots, \nu_{N}=e^{i\left(\Re-\mathfrak{w}_{1} \nu_{1}-\mathfrak{w}_{2} \nu_{2}-\ldots-\mathfrak{w}_{N} \nu_{N}\right) \Re} \\
\cdot u_{\nu_{1}, \ldots, \nu_{N}}\left(\Re, \mathfrak{r}_{i}-\mathfrak{r}_{j}\right),
\end{aligned}
$$

worin $u_{\nu_{1}, \ldots, \nu_{N}}$ zunächst noch ganz beliebig sein kann. $\Re$ ist darin die Schwerpunktskoordinate der Elektronen, $\mathfrak{r}_{i}-\mathfrak{r}_{j}$ sind deren Relativkoordinaten. Beim Einsetzen von (13) in (12) erhalten wir

$$
u_{\nu_{1}, \ldots, \nu_{N}}\left(\Re+\mathfrak{a}, \mathfrak{r}_{i}-\mathfrak{r}_{j}\right)=u_{\nu_{1}, \ldots, \nu_{N}}\left(\Re, \mathfrak{r}_{i}-\mathfrak{r}_{j}\right) ;
$$

$u$ ist also invariant gegenüber einer Verschiebung von $\Re$ um einen Gittervektor.

Wir erhalten somit als Ergebnis ${ }^{7}$ :

Die Lösungen der Gl. (7) haben die Form

$$
e^{i \Re \Re} \sum_{(v)} e^{-i\left(\mathfrak{w}, v_{1}+. .+\mathfrak{w}_{N} v_{N}\right) \Re} u_{\nu_{1} \ldots, \nu_{N}}\left(\Re, \mathfrak{r}_{i}-\mathfrak{r}_{j}\right)\left(b_{1}^{*}\right)^{\nu_{1}} \ldots\left(b_{N}^{*}\right)^{\nu_{N}} \varphi_{0}=e^{i \Re \Re} U_{\Re}\left(\Re, \mathfrak{r}_{i}-\mathfrak{r}_{j} ; b_{\lambda}^{*}\right) .
$$

Darin sind die $u_{\nu_{1}}, \ldots, \nu_{N}$ in der Schwerpunktskoordinate $\Re$ gitterperiodische Funktionen, die noch in

${ }^{6}$ Diese Eigenfunktionen sind noch nicht normiert, was für das folgende aber nicht wesentlich ist.

7 Wir denken uns dazu die eben durchgeführten Schlüsse für alle drei $\mathfrak{a}_{i}$ ausgeführt. Dies ist ohne wei-

allgemeiner Weise von den Relativkoordinaten $\mathfrak{r}_{i}-\mathfrak{r}_{j}$ und evtl. noch von Spinkoordinaten (bzw.

teres möglich, da die Matrizen $\left(c_{i j}\right)$ in Gl. (10), die für verschiedene $\mathfrak{a}_{i}$ verschieden sein können, sich simultan auf Hauptachsen bringen lassen. 
von Spinorindizes) abhängen. $U_{\Re}$ hängt natürlich nicht nur von $\Re$, sondern auch noch von weiteren Quantenzahlen ab. Dies deuten wir gelegentlich durch einen weiteren Index $J$ an.

Die endgültige Form (15) der Lösung ist leicht einer einfachen Deutung zugänglich. Dazu denken wir uns die $u_{v_{1}}, \ldots, v_{v_{N}}$ in der Schwerpunktskoordinate in eine Fourier-Reihe über eine Gitterzelle entwickelt. Die einzelnen Reihenglieder sind dann Eigenfunktionen des Gesamtimpulses freier Elektronen und zwar treten nur solche Impulse auf, die (bis auf den Faktor $\hbar$ ) Vektoren $\mathfrak{b}_{\kappa}$ des reziproken Gitters sind. Nehmen wir zu diesen Funktionen $e^{i \mathfrak{b}_{k} \Re}$ noch den Faktor $e^{i\left(\Re-\mathfrak{w}_{1} \nu_{1} \ldots-\mathfrak{w}_{N} \nu_{N}\right) \Re}$ hinzu und bedenken, daß zu der Eigenfunktion $\left(b_{1}^{*}\right)^{\nu_{1}} . .\left(b_{N}^{*}\right)^{\nu_{N}} \varphi_{0}$ der Impuls $\sum_{\lambda} \hbar \mathfrak{w}_{\lambda} v_{\lambda}$ gehört, so können wir sagen:

In (15) sind nur solche Funktionen kombiniert, die zum gleichen Gesamtimpuls von Elektronen und Oszillatoren gehören oder zu einem, der sich davon nur um einen (mit $\hbar$ multiplizierten) Vektor $\mathfrak{b}_{w}$ des reziproken Gitters unterscheidet. Unter dem letzteren Fall sind insbesondere die in der Theorie der metallischen Leitung bei tiefen Temperaturen auftretenden und dort wesentlichen Umklapp-Prozesse enthalten.

Bei Spezialisierung unserer Voraussetzungen über den Hamilton-Operator (7) erhalten wir einige aus der Literatur bekannte Resultate:

Für Elektronen im ruhenden Gitter ist jedes Glied der Reihe (15) für sich Eigenfunktion; die Lösung der Schrödinger-Gleichung ist also in diesem Falle von der Gestalt

$$
e^{i \Re \Re} U_{\Re}\left(\Re, \mathfrak{r}_{i}-\mathfrak{r}_{j}\right),
$$

worin $U_{\Re}$ in der Schwerpunktskoordinate $\Re$ der Elektronen periodisch ist. Im Falle einer abstandsabhängigen Wechselwirkung wurde diese Lösungsform schon von $\mathrm{Volz}^{1}$ hergeleitet. Bei einem Elektron reduziert sich der Satz von Volz auf das Theorem von Bloch ${ }^{8}$, demzufolge die Lösung die Form

$$
e^{i \mathfrak{f r}} u_{\mathfrak{f}}(\mathfrak{r})
$$

hat, worin $u_{\mathfrak{f}}$ in der Elektronenkoordinate $\mathfrak{r}$ gitterperiodisch ist.

Für ein einzelnes Elektron im schwingenden Gitter lautet hingegen die Form der Lösung

$$
\begin{aligned}
& e^{i \mathfrak{f r}} \sum_{(v)} e^{-i\left(\mathfrak{w}_{1} \nu_{1}+\ldots+\mathfrak{w}_{N} v_{N}\right) \mathfrak{r}} \\
& \cdot u_{\nu_{1}, \ldots, \nu_{N}}(\mathfrak{r})\left(b_{1}^{*}\right)^{\nu_{1}} \ldots\left(b_{N}^{*}\right)^{\nu_{N}} \varphi_{0} .
\end{aligned}
$$

${ }^{8}$ F. Bloch, Z. Phys. 52, 555 [1928].

${ }^{9}$ H. Fröhlich, H. Pelzer u. S. Zienau, Phil. Mag. 41, 221 [1950].
Ist insbesondere der Hamilton-Operator invariant gegenüber einer infinitesimalen Transformation der Elektronenkoordinaten und der Schallquantenoperatoren, so sind die $u_{v_{1}, \ldots, v_{N}}$ Konstanten. Bei der Untersuchung der Bewegung einzelner Elektronen in einem schwingungsfähigen polaren Medium wurden Fröhlich, Pelzer und Zienau ${ }^{9}$ auf einen solchen Fall geführt. Wie wir den eben im Anschluß an (15) durchgeführten Betrachtungen entnehmen können, treten bei diesem von Fröhlich ${ }^{10}$ (implizit auch in seiner Supraleitungstheorie ${ }^{11}$ ) verwendeten Wechselwirkungsansatz keine Umklapp-Prozesse auf.

\section{Einige allgemeine Eigenschaften der Lösung}

a) Die Abhängigkeit von $\Re$

Wir zeigen, daß $\mathfrak{\AA}$ auf den Bereich $-\pi / a_{i}<k_{i} \leq \pi / a_{i}$, $a_{i}=\left|\mathfrak{a}_{i}\right|$ reduziert werden kann. Dazu genügt der Nachweis, daß die Form der Lösung erhalten bleibt, wenn $\mathfrak{K}$ durch $\mathfrak{K}^{\prime}$ mit $\mathfrak{K}=\mathfrak{K}^{\prime}+\mathfrak{b}$ (worin $\mathfrak{b}$ ein Vektor des reziproken Gitters ist) ersetzt wird. Beim Einsetzen von $\mathfrak{\aleph}^{\prime}+\mathfrak{b}$ in (15) ergibt sich

$$
\begin{aligned}
e^{i\left(\Re^{\prime}+\mathfrak{b}\right) \Re} \sum_{(\nu)}\left[e^{-i\left(\mathfrak{w}_{1} \nu_{1}+\ldots+\mathfrak{w}_{N} \nu_{N}\right) \Re}\right. \\
\left.\cdot u_{\nu_{1}, \ldots, \nu_{N}}\left(\Re, \mathfrak{r}_{i}-\mathfrak{r}_{j}\right)\left(b_{1}^{*}\right)^{\nu_{1}} \ldots\left(b_{N}^{*}\right)^{\nu_{N}} \varphi_{0}\right],
\end{aligned}
$$

was sich auch in der Form

$$
\begin{aligned}
e^{i \Re^{\prime} \Re} & \sum_{(\nu)}\left[e^{-i\left(\mathfrak{w}_{1} \nu_{1}+\ldots+\mathfrak{w}_{N} \nu_{N}\right) \Re}\right. \\
& \cdot\left\{e^{i 6 \Re} u_{\nu_{1}, \ldots \nu_{N}}\left(\Re, \mathfrak{r}_{i}-\mathfrak{r}_{j}\right)\right\}\left(b_{1}^{*}\right)^{\nu_{1}} \ldots\left(b_{N}^{*}\right)^{\nu_{N}} \varphi_{0}
\end{aligned}
$$

schreiben läßt. Da

$$
e^{i ø \Re} u_{\nu_{1}, \ldots, \nu_{N}}\left(\Re, \mathfrak{r}_{i}-\mathfrak{r}_{j}\right)
$$

wieder eine in $\Re$ gitterperiodische Funktion ist, ist auch (16) wieder eine ,Normalform“ (15).

Durch die Forderung, daß die Wellenfunktion in jeder einzelnen Elektronenkoordinate periodisch mit dem Grundgebiet sein soll, werden ähnlich wie bei einem Elektron im Bändermodell bestimmte $\mathfrak{R}$-Werte ausgesondert: Verschiebt man alle Elektronenkoordinaten gleichzeitig um einen der das Grundgebiet aufspannenden Vektoren, so gehen die in der Summe (15) stehenden $u_{v_{1}}, \ldots v_{N}$ wegen ihrer Periodizitätseigenschaft in $\Re$ in sich über, ebenso aber auch die unter der Summe dabeistehenden Exponentialfaktoren, da die in ihnen auftretenden Wellenlängen der Gitterschwingungen im Grundgebiet aufgehen sollten. Aus dem vor der Summe stehenden Faktor $e^{i \Re \Re}$ ist hingegen $e^{i \Re \Re+i \Re \mathscr{G}_{j}}$ geworden. Damit auch dieser

${ }^{10}$ H. Fröhlich, Proc. Roy. Soc. A, 160, 230 [1937].

${ }^{11}$ H. Fröhlich, Phys. Rev. 79, 845 [1950]. 
Faktor invariant gegenüber der eben betrachteten Verschiebung ist, muß daher gelten:

$$
\mathfrak{\Re}=\left(\frac{2 \pi \varkappa_{1}}{G_{1}}, \frac{2 \pi \varkappa_{2}}{G_{2}}, \frac{2 \pi \varkappa_{3}}{G_{3}}\right) ; \quad \varkappa_{i} \text { ganze Zahlen. }
$$

\section{b) Die Orthogonalität}

Da der Hamilton-Operator hermitesch ist, sind die zu verschiedenen Eigenwerten gehörigen Lösungen aufeinander orthogonal. Ferner sind die zu verschiedenen (reduzierten) $\mathfrak{\Re}$ gehörigen Lösungen aufeinander orthogonal, was wie folgt bewiesen wird:

Für die Funktionen $\psi_{\Re_{1}}$ und $\psi_{\mathfrak{N}_{2}}$ in

$$
\left(\int \psi_{\Re_{1}}^{*} \psi_{\Omega_{2}} \mathrm{~d} \tau\right)
$$

setzen wir die Entwicklung (15) ein

$$
\begin{aligned}
\sum_{(\nu)} \sum_{(\mu)} \int\left\{e^{-i\left[\Re_{1}-\left(\mathfrak{w}_{1} \nu_{1}+\ldots\right)\right] \Re} u_{\nu_{1}}^{*} \ldots \cdot e^{i\left(\Re_{2}-\left(\mathfrak{w}_{1} \mu_{1}+\ldots\right)\right) \Re}\right. \\
\left.\cdot u_{\mu_{1}, .} \mathrm{d} \tau\left(\varphi_{0}, b_{N}^{\nu_{N}} \ldots b_{1}{ }^{\nu_{1}}\left(b_{1}^{*}\right)^{\mu_{1}} \ldots\left(b_{N}^{*}\right)^{\mu_{N}} \varphi_{0}\right)\right\} .
\end{aligned}
$$

Wegen der Orthogonalität verschiedener Eigenfunktionen $\left(b_{1}^{*}\right)^{\nu_{1}} \ldots\left(b_{N}^{*}\right)^{\nu_{N}} \varphi_{0}$ bleibt stehen ${ }^{12}$ :

$\sum_{(\nu)} \int e^{-i\left(\Omega_{1}-\Omega_{2}\right)} u_{\nu_{1}, \ldots, v_{N}}^{*} \cdot u_{\nu_{1}, \ldots, v_{N}} \mathrm{~d} \tau \cdot v_{1} ! v_{2} ! \ldots v_{N} !$.

Wie früher ${ }^{1}$ bewiesen wurde, verschwinden sämtliche Summanden

$\int e^{-i\left(\Re_{1}-\Re_{2}\right) \Re} u_{\nu_{1}, \ldots,{ }_{N}}^{{ }} \cdot u_{\nu_{1}, \ldots,{ }_{N}} \mathrm{~d} \tau \operatorname{mit} \Re_{1} \neq \Re_{2}\left(\mathfrak{b}_{i}\right)$

für sich. Damit ist der Beweis für die Orthogonalität von Wellenfunktionen mit verschiedenem $\mathfrak{\AA}$ erbracht.

Es bleibt noch die Frage nach der Orthogonalität von Lösungen zu untersuchen, die zu gleichem $E$ und $\Re$ gehören. Falls diese Funktionen noch nicht orthogonal sein sollten, so können wir sie dadurch orthogonal machen, daß wir die $U_{\Re}$ unter sich nach einem der bekannten Orthogonalisierungsverfahren orthogonalisieren. Dabei bleibt offensichtlich die Form der Lösung (15) erhalten.

\section{c) Die Symmetrie}

Alle unsere Ausführungen gelten unabhängig von Symmetrie-Eigenschaften der Wellenfunktion. Wenn wir uns auf das physikalische System von Elektronen beziehen wollen, so müssen wir natürlich verlangen, daß die Wellenfunktionen in den Orts- und Spinkoordinaten der Elektronen antisymmetrisch sind. Diese Symmetrieforderung ${ }^{13}$ geht dann unmittelbar in die Entwicklungskoeffizienten $u_{v_{1}}, \ldots, v_{N}$ ein.

12 Der Faktor $v_{1} ! v_{2} ! \ldots v_{N}$ ! tritt hier auf, weil die $\left(b_{1}^{*}\right)^{\nu_{1}} \ldots\left(b_{N}^{*}\right)^{\nu} N \varphi_{0}$ nicht normiert sind.

${ }_{13}$ Für die Frage, wie sich diese Forderung bei Funktionen auswirkt, die nur von Ortskoordinaten ab-
Die der Bose-Statistik entsprechende Symmetrie für die Schallquanten ist schon durch die Benutzung der Erzeugungs- und Vernichtungsoperatoren (mit der Minus-Vertauschungsrelation) berücksichtigt.

\section{Der Ausdruck für den Strom}

Die zeitliche Verschiebung des Schwerpunkts eines Elektrons $\dot{\overline{\mathfrak{r}}}_{i}$ ist nach der Rechenvorschrift der Quantenmechanik durch

$$
\dot{\overline{\mathfrak{r}}}_{i}=\frac{i}{\hbar} \overline{\left(\mathscr{H} \mathfrak{r}_{i}-\mathfrak{r}_{i} \mathscr{H}\right)}
$$

gegeben, wobei der Mittelungsstrich die Bildung des Erwartungswertes bedeutet. Der Strom aller Elektronen, gemittelt über das Grundgebiet, wird dann durch

$$
\text { e } \Sigma \dot{\overline{\mathfrak{r}_{i}}}=\frac{i}{\hbar} \text { en } \overline{(\mathscr{H} \Re-\Re \mathscr{H})}
$$

dargestellt. $n$ ist die Zahl der Elektronen im Grundgebiet.

Zur Herleitung eines Zusammenhangs zwischen dem Gesamtstrom und der Energie gehen wir mit dem Ansatz

$$
\psi_{\Re}=e^{i \Re \Re} U_{\Re}\left(\Re, \mathfrak{r}_{i}-\mathfrak{r}_{j} ; b_{\lambda}^{*}\right)
$$

in die Gleichung

$$
\left(\mathscr{H}-E_{\Re}\right) \psi_{\Re}=0
$$

ein, differenzieren nach $\Re$ und multiplizieren mit $\psi_{\Omega}^{*}$. Bei der Integration über das Grundgebiet wird dann

$$
\begin{gathered}
-\operatorname{grad}_{\Re} E_{\Re} \int \psi_{\Re}^{*} \psi_{\Re} \mathrm{d} \tau+\int \psi_{\Re}^{*}\left(\mathscr{H}-E_{\Re}\right) i \Re \psi_{\Re} \mathrm{d} \tau \\
+\int \psi_{\Re}^{*}\left(\mathscr{H}-E_{\Re}\right) e^{i \Re \Re} \operatorname{grad}_{\Re} U_{\Re} \mathrm{d} \tau=0 .
\end{gathered}
$$

Wegen der Normierung ist das erste Integral gleich 1. Im zweiten schreiben wir statt $E_{\Re} \psi_{\Re}$ gemäß (18) wieder $\mathscr{H} \psi_{\Re}$ und erhalten dann für dieses Integral

$$
i \int \psi_{\Re}^{*}(\mathscr{H} \Re-\Re \mathscr{H}) \psi_{\Re} \mathrm{d} \tau .
$$

Beim dritten Integral bekommen wir, da $\mathscr{H}$ ein hermitescher Operator ist,

$$
\int e^{i \Re \Re} \operatorname{grad}_{\Re} U_{\Re}\left\{\left(\mathscr{H}^{*}-E_{\Re}\right) \psi_{\Re}\right\} \mathrm{d} \tau .
$$

Der in geschweifte Klammern gesetzte Ausdruck ist gerade die konjugiert-komplexe linke Seite von Gl. (18), verschwindet also. Unter Beachtung, daß (20) bis auf den Faktor $1 / \hbar$ den Strom darstellt, bekommen wir somit als Endresultat

hängig angesetzt werden, sei auf eine Arbeit von A. Brickstock u. J. A. Pople, Phil. Mag. 44, 697 [1953], verwiesen. 


$$
\subseteq=\frac{e n}{\hbar} \operatorname{grad}_{\Omega} E_{\Omega} .
$$

Als Spezialfälle nennen wir die von Volz und Haken $^{1}$ angegebene Geschwindigkeitsformel für Elektronen mit Coulombscher Wechselwirkung im ruhenden Gitter, die sich für ein Elektron auf die Formel von Peierls ${ }^{14}$ reduziert, sowie den von Fröhlich, Pelzer und Ziena ${ }^{9}$ behandelten Fall eines Elektrons in Wechselwirkung mit Oszillatoren in polaren Medien.

Um einen Einblick in die „Wirkungsweise“ von Formel (21) zu erhalten, mag es nützlich sein, diese Formel auf den Spezialfall von Elektronen ohne Wechselwirkung anzuwenden. Wir erläutern dies am Beispiel von. zwei Elektronen mit den Wellenzahlen $k_{1}, k_{2}$, die sich etwa in einem ruhenden, eindimensionalen Gitter bewegen sollen. In diesem Falle ist $E$ einfach eine Summe aus den beiden Einzelenergien $E_{0}\left(k_{1}\right)$ und $E_{0}\left(k_{2}\right)$. Als neue Quantenzahlen haben wir $K=k_{1}+k_{2}$ zu nehmen, als weitere kommt im wesentlichen nur $J=k_{1}-k_{2}$ in Frage ${ }^{15}$.

Wir bilden nun

$$
S=\frac{2 e}{\hbar} \frac{\partial E}{\partial K}=\frac{2 e}{\hbar}\left(\frac{\partial E_{0}}{\partial k_{1}} \frac{\partial k_{1}}{\partial K}+\frac{\partial E_{0}}{\partial k_{2}} \frac{\partial k_{2}}{\partial K}\right)
$$

und erhalten wegen $k_{1}=1 / 2(K+J), k_{2}=1 / 2(K-J)$ :

$$
S=\frac{e}{\hbar}\left(\frac{\partial E_{0}}{\partial k_{1}}+\frac{\partial E_{0}}{\partial k_{2}}\right) \text {. }
$$

Die Benutzung der Geschwindigkeitsformel für ein Elektron

ergibt als Endresultat

$$
v_{i}=\frac{1}{\hbar} \frac{\partial E_{0}}{\partial k_{i}}
$$

$$
S=e\left(v_{1}+v_{2}\right) .
$$

5. Folgerungen aus dem Ausdruck für den Strom

\section{Der Blochsche Satz}

Gl. (21) gestattet es, den Strom allein aus dem Energieverlauf zu berechnen, ohne daß man die Wellenfunktionen zu kennen braucht. Dies ist natürlich eine Vereinfachung, da im allgemeinen die Energie leichter zu berechnen ist als die zugehörige Wel-

14 R. Peierls, Z. Phys. 53, 255 [1929].

15 Außerdem wäre hier noch diejenige Quantenzahl zu nennen, die die Nummer des Bandes angibt, in dem sich die Elektronen befinden. Diese ist hier aber unwichtig.

${ }^{16}$ F. Bloch, Anhang zu L. Brillouin, J. Phys. Radium 4, 334 [1933]. Der Blochsche Satz ist bekanntlich (s. lenfunktion. Wie außerdem das Beispiel des Bändermodells, bei dem Gl. (21) vielfach verwendet wird, zeigt, genügt dann für viele Diskussionen auch schon ein qualitatives Verständnis des Verlaufs der Energiekurve in Abhängigkeit von $\mathfrak{\Re}$. Bei der großen Zahl der betrachteten Elektronen haben wir selbst im eindimensionalen Fall natürlich nicht eine einfache Kurve wie bei einem Elektron, sondern eine hochdimensionale Fläche vor uns. Über die Gestalt dieser Fläche, die also die funktionelle Abhängigkeit der Energie von der Quantenzahl $\mathfrak{\Re}$ und weiteren Quantenzahlen $J$ wiedergibt, können nur ausführliche Untersuchungen an Modellbeispielen Auskunft geben. Überhaupt erscheint uns die Auffindung von näherungsweisen Quantenzahlen, die das System in geeigneter Weise beschreiben, als eines der wichtigsten, zugleich aber auch schwierigsten Probleme in der Theorie des Mehrelektronenproblems im Festkörper. In unseren Diskussionen wird indessen nur der Verlauf des Schnittes durch die Energiefläche parallel zur $\mathfrak{\AA}$-,,Achse“", also bei festgehaltenen Quantenzahlen $J$, vorkommen.

Wie schon vom Bändermodell her geläufig ist, tritt dieses $\mathfrak{\Re}$ in gewissem Sinne an die Stelle des Impulses. Ähnlich wie man beim freien Elektron durch Variation des Impulses einen energetisch tiefsten Zustand erreichen kann, so werden wir auch hier, wenn wir $\mathfrak{\jmath}$ laufen lassen, zu einem energetisch tiefsten, also stabilen, evtl. aber auch zu einem metastabilen Zustand (Beispiel: Bändermodell) gelangen. Für solche Zustände ist notwendig $\operatorname{grad}_{\mathfrak{K}} E$ $=0$. Wegen Gl. (21) erhalten wir daraus unmittelbar den von Bloch ${ }^{16}$ aufgestellten Satz, daß der energetisch tiefste Zustand des Festkörpers stromlos ist. Der Gültigkeitsbereich unseres Beweises ist durch den Gültigkeitsbereich des anfänglich angenommenen Hamilton-Operators festgelegt. Durch diesen Hamilton-Operator werden wohl alle für den Festkörper in Frage kommenden Arten von Wechselwirkungen zwischen den Elektronen (einschließlich der Spin-Bahn-Wechselwirkung ${ }^{17}$ ) erfaßt, so daß der Beweis in dieser Hinsicht volle Allgemeinheit beanspruchen darf. Bei der Berücksichtigung der Kernbewegung, also der Schwingun-

etwa: F. London, Superfluids, Bd. 1, Verlag John Wiley \& Sons, New York 1950) für die Entwicklung einer atomistischen Theorie der Supraleitung von Bedeutung, da durch ihn die Versuche widerlegt werden, die Supraleitung durch die Annahme spontaner, stromtragender Zustände, die energetisch günstiger als stromlose Zustände sein sollen, zu erklären. 
gen des Gitters, hatten wir hingegen von der in der bisherigen Theorie des Festkörpers üblichen Näherung Gebrauch gemacht, daß die Schwingungen harmonisch und zwar mit einer vom Elektronenzustand unabhängigen Frequenz erfolgen. Solange wir jedoch nicht zu sehr hohen Anregungsstufen des Gesamtsystems übergehen und außerdem die betrachtete Quantenzahl $\Re$, also auch den Gesamtzustand nur in einem kleinen Bereich variieren, haben wir allen Grund zu der Annahme, daß auch diese Konzeption gerechtfertigt ist.

Es bleibt schließlich noch der Fall zu erledigen, daß in die Formulierung des Blochschen Satzes der Temperaturbegriff hineingebracht wird. Dann läßt sich zeigen, daß Zustände mit einem Minimum der freien Energie stromlos sind. Nach B ohm ${ }^{17}$ genügt dazu der Nachweis, daß es zu jedem stromtragenden Zustand einen benachbarten mit geringerer (innerer) Energie gibt. Dies folgt aber bei uns sofort aus Gl. (21).

\section{Schlußbemerkungen}

Obgleich sich bezüglich der Form der Wellenfunktion und des Geschwindigkeitsgesetzes eine formal recht enge Analogie zu den entsprechenden, sich auf ein Elektron beziehenden Formeln des Bändermodells ergibt, so sind die Vorstellungen, die dieser Arbeit zugrunde liegen, doch grundverschieden von denen des Bändermodells. Wir heben hier besonders die Unterschiede in der Behandlung der Wechselwirkung zwischen den Elektronen und Gitterschwingungen hervor. Im Bändermodell werden

17 Insofern und wegen der Berücksichtigung der Wechselwirkung der Elektronen mit den Gitterschwingungen geht dieser Beweis wesentlich über den bisher einzigen ausführlichen Beweis von D. Bohm, Phys. Rev. 75, 502 [1949] hinaus, in welchem die Coulombsche und die magnetische Wechselwirkung die Elektronen aus dem Gesamtsystem herausgelöst und ihre Kopplung mit den Gitterschwingungen dann nachträglich störungstheoretisch behandelt. Dementsprechend wird die Wirkung eines äußeren elektrischen Feldes durch die Änderung des $\mathrm{Zu}$ stands der Elektronen wiedergegeben, während etwa der Temperaturzustand im wesentlichen durch die Bose-Einstein-Verteilung der Schallquanten erfaßt wird.

In der vorliegenden Untersuchung hingegen wird das System aus Elektronen und Gitterschwingungen als ein quantenmechanisches Gesamtsystem behandelt, so daß dann nur mehr von seinen stationären Zuständen gesprochen werden kann. Die Einwirkung eines elektrischen Feldes oder das Vorhandensein eines Temperaturzustandes müssen in unserem Falle also unter Zugrundelegung des Gesamtsystems behandelt werden. Unter der Wirkung des elektrischen Feldes durchläuft das Gesamtsystem eine Folge immer höherer Anregungszustände. Ein Gleichgewichtszustand ist erst dann zu erhalten, wenn auch die Energieabgabe nach außen mit in die Untersuchung einbezogen wird. Das bedeutet, daß eine ganz neue und gegenüber der bisherigen wesentlich umfassendere Theorie der Leitfähigkeit aufgestellt werden muß. Einen ersten Ansatzpunkt für eine solche Neuformulierung bieten Untersuchungen an Modellen, wie in nachfolgenden Arbeiten gezeigt werden soll.

Herrn Prof. Dr. H. Volz danke ich für mehrere wertvolle Ratschläge zur Abfassung der Arbeit.

zwischen Elektronen im ruhenden Gitter berücksichtigt werden. Weitere - nach unserem Erachten nicht ganz ausreichende - Beweise dieses Satzes finden sich bei Bloch ${ }^{16}$; H. G. Smith u. J. O. Wilhelms, Rev. Mod. Phys. 7, 266 [1935]; L. Brillouin, Proc. Roy. Soc. A 152, 19 [1935]. 